\title{
Workshop CPS et E-Health
}

La stratégie E-Health de la FMH et le projet de carte de professionnel de santé (CPS) ont suscité un grand intérêt: plus de trente sociétés médicales ont répondu à notre invitation à un workshop et ont envoyé le 3 septembre 2008 leurs délégués à Berne pour obtenir des informations du groupe de projet, mais aussi des réponses à leurs questions.

L'atelier a été introduit par le Dr Monique Gauthey, nouvelle responsable du domaine eHealth au sein du Comité central de la FMH; elle a présenté un exposé sur le développement actuel en Europe et en Suisse du domaine de la cybersanté et de la carte de professionnel de santé (cf. éditorial).

Au cours de la première partie de l'atelier, le Dr Max Giger, membre du Comité central, a présenté les avantages et les possibilités d'utilisation de la CPS. Les M. Georg Kassowitz et Harald Heuser ont donné ensuite des informations sur le soutien opérationnel et technique. Au cours de la seconde partie, Mme Judith Wagner, res- ponsable de la cybersanté au sein de la FMH, a renseigné les participants sur les développements importants du domaine E-Health pour la FMH.

Après discussion, il est clairement ressorti de cet atelier que la HPC et la cybersanté marqueront à l'avenir fortement l'activité quotidienne des médecins. Il est d'autant plus important que le corps médical soit tenu régulièrement au courant de ce domaine. C'est ainsi que les intervenants ont résumé leurs exposés pour le lectorat du BMS. Vous trouverez leurs articles dans les pages qui suivent.
Dr Max Giger, membre du Comité central de la FMH

\section{Mise en place et utilité de la CPS}

La carte de professionnel de santé (CPS) a une fonction de carte d'identité pour l'ensemble des médecins exerçant en Suisse, et leur confère une authentification à la fois physique et virtuelle: cela permet de signer électroniquement des documents, et ainsi de les envoyer et de les recevoir d'une manière sécurisée. Grâce à sa structure ouverte, la CPS peut être utilisée dans le domaine de la cybersanté que ce soit au niveau national ou international. Elle garantit aussi une interopérabilité, c'est-à-dire qu'elle permet aux médecins et à d'autres groupes de professionnels de la santé, de procéder à un tel échange électronique de données. Les destinataires peuvent utiliser ces informations comme s'ils les avaient générées eux-mêmes. A titre d'exemple, le médecin pourra directement reporter dans ses propres dossiers médicaux électroniques les données vitales, les résultats de laboratoires et les rapports des médecins traitants. La CPS est en outre compatible avec la carte d'assuré qui sera vraisemblablement remise aux patients avant le $1^{\mathrm{er}}$ janvier 2010 par les assureurs-maladie.
Par le biais de la carte de professionnel, la FMH se positionne comme un partenaire important dans le domaine de la cybersanté. Les décisions stratégiques de la Chambre médicale de décembre 2005 et 2006 fondent ainsi une étape essentielle pour la médecine de demain. Suite à un appel d'offre conforme aux directives de l'OMC, les premières étapes avec Swisscom IT Services ont été réalisées en août 2007. Les travaux liés aux applications-pilote ont pour leur part débuté en juillet 2008. La livraison de la CPS aux membres de la FMH et à d'autres médecins est prévue pour 2009. L'ensemble des membres de la FMH recevra ensuite les documents nécessaires pour l'enregistrement.

\section{Possibilités d'utilisation}

La CPS permettra un échange sécurisé de données avec myFMH, par exemple pour modifier ses données personnelles. Les structures informatiques nécessaires pourraient être fournies aux sociétés de discipline médicale et à leurs membres, pour leur permettre d'utiliser la carte 
pour enregistrer leurs sessions de formation continue. La comptabilisation des crédits de formation continue et la délivrance consécutive des certificats s'en trouveraient facilitées. Les médecins en formation postgraduée pourraient répertorier directement dans leur logbook les formations suivies, et les établissements de formation pourraient signer électroniquement les certificats délivrés. Afin d'intégrer les principaux logiciels de cabinets médicaux, l'utilisation de la carte en collaboration avec le Swiss Medical Suite est actuellement examinée. Last but not least, l'utilisation d'arbres décisionnels (Decision Support Systems) dans le cadre de la prescription électronique de médicaments contribuerait à accroître la sécurité des prescriptions.

Ces différentes possibilités d'utilisation de la CPS sont autant d'illustrations de l'objectif que nous nous fixons: une communication sécurisée et efficace tout au long de la trajectoire du patient, qui permettra au médecin un gain de temps précieux pour accéder aux documents médicaux, mais aussi un gain de temps précieux pour les patients.

\section{Harald Heuser, membre du groupe de projet} CPS-FMH

\section{Sécurité des données et aspects techniques de la CPS-FMH}

\section{Champs d'application et technologie}

Avec la carte de professionnel de santé, la FMH dispose d'un composant clé basé sur des standards internationaux, pour les trois applications fondamentales suivantes :

- la création de signatures électroniques;

- l'authentification de personnes dans des systèmes électroniques;

- le cryptage de données lors de leur transmission ou de leur archivage.

Lors de l'utilisation de la CPS, on utilise les techniques de cryptage conformes à la procédure «clé privée/clé publique». Cette procédure est utilisée à l'échelle internationale et est soutenue par de nombreuses applications logicielles standards. Dans un environnement sécurisé, on crée une paire de clés cryptées, composée d'une clé privée et d'une clé publique. La clé privée est placée exclusivement sur la carte, alors que la clé publique est publiée comme certificat dans un service Web, qui assurera son suivi.

\section{Conditions d'utilisation}

Pour utiliser la CPS-FMH, il faut disposer d'un ordinateur traditionnel avec un système d'exploitation courant (Windows, Mac ou Linux), d'un lecteur de cartes standard, d'un logiciel pilote CPS-FMH et d'un logiciel qui fonctionne avec les applications standards mentionnées cidessus. Des exemples pour de tels logiciels sont Outlook ou Acrobat Professional.

Un Software Development Kit (SDK) est disponible pour utiliser la CPS en liaison avec des applications spécifiques (systèmes de gestion du cabinet médical). Il permet au fabricant concerné d'intégrer très rapidement l'utilisation de la carte dans telle ou telle application spécifique.

\section{Sécurité}

La CPS de la FMH existe en plusieurs versions avec divers certificats qui se distinguent par des niveaux de sécurité différents, avec d'autres effets juridiques. Selon le niveau de sécurité souhaité, on installe le certificat nécessaire (ou même plusieurs certificats) sur la carte. Le niveau maximum permet même de créer des signatures assimilées à des signatures manuelles par la législation suisse, niveau que nous avons pu atteindre seulement en choisissant Swisscom IT Services en qualité de fournisseur de la CPS. En effet, il n'existe en Suisse que quatre entreprises certifiées pour répondre aux exigences de sécurité élevées de la loi sur la signature électronique.

\section{Etablissement et contrôle des signatures électroniques}

Avec la CPS-FMH, la signature électronique est créée en deux étapes: tout d'abord, on établit une «valeur de hachage» (Hash-Wert) du fichier ou du message concerné. La valeur de hachage d'un fichier ou d'un message correspond aux empreintes digitales d'une personne. Ensuite, la valeur de hachage est cryptée avec la clé privée du signataire et attachée au fichier ou au message. Le certificat public du signataire y est également attaché.

La vérification de la valeur de hachage permet aussi d'établir après coup de manière incontestable, si le fichier ou le message est intact ou s'il a été modifié. La vérification du certificat 
public permet quant à elle d'établir de manière certaine l'identité du signataire et la validité de sa signature. Celle-ci peut être prolongée si l'on signe à nouveau le fichier ou le message signé avec un certificat d'une durée de validité plus étendue.

\section{Cryptage et décryptage}

Le fichier ou le message à crypter le sera au moyen du certificat officiel du destinataire prévu, mis à disposition du destinataire au moyen d'une connexion librement choisie. Le fichier ou le message crypté ne peut être rendu lisible que par le destinataire concerné, au moyen de son propre certificat privé. Cela garantit que le fichier ou le message restera confidentiel envers tous, à partir du moment et du lieu de son cryptage par l'expéditeur jusqu'au moment et au lieu de son décryptage par le destinataire.
Un fichier ou un message crypté peut se comparer à une lettre scellée transmise par un messager à son destinataire, après que celui-ci se soit identifié en présentant une pièce d'identité. Un fichier ou un message non crypté peut se comparer à une carte postale.

\section{Perspectives}

La CPS -FMH permet de disposer des applications de base pour un échange flexible et malgré tout sécurisé des informations dans le système de santé. La CPS aura aussi un effet de catalyseur pour réaliser des applications basées sur la carte, permettant d'alléger de différentes manières le travail quotidien dans le système de santé. La FMH soutient clairement de telles applications et entreprendra encore dans le cadre de la période pilote de nouvelles démarches dans ce sens.
Georg Kassowitz, chef de projet CPS-FMH
En introduisant une carte de professionnel de santé (CPS ou HPC) aux dimensions d'une carte de crédit, la FMH propose aux médecins un outil pour se raccorder à des supports électroniques et exercer leur activité quotidienne en accord avec la technique moderne. La FMH a lancé le projet pour s'assurer que cette entreprise complexe

Figure 1

Aperçu des différentes phases du projet.

\section{Aperçu des différentes phases du projet}

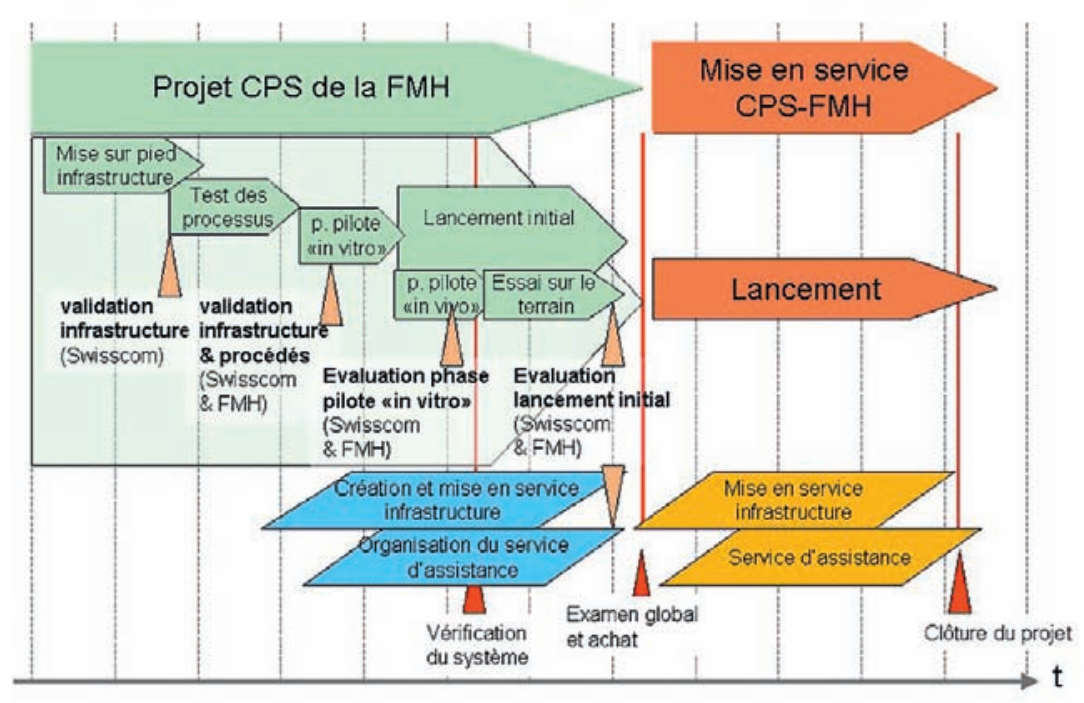

soit coordonnée et harmonisée de la meillleure façon.

Ce projet a été structuré avec plusieurs phases et coordonné d'entente avec le fournisseur (Swisscom IT Services). Après la mise au point de l'infrastructure, la CPS sera soumise à des premiers tests pratiques auprès d'un cercle limité d'utilisateurs. Bien que les techniques utilisées fassent appel à des technologies bien connues et éprouvées, des problèmes pourraient survenir dans l'interaction entre les différents composants. S'ils devaient se produire, ils seraient rapidement résolus avec sûreté. Dans une deuxième phase pilote, la CPS sera remise à un échantillon d'utilisateurs représentant au mieux toutes les exigences possibles. Les tests, essais et collectes d'expériences permettront de combler les lacunes ou les problèmes qui pourraient alors se faire jour.

La CPS ne sera remise à l'ensemble des médecins qu'après l'achèvement fructueux de phases pilotes axées sur la pratique. La procédure d'enregistrement en vue d'obtenir une carte avec une qualité d'utilisateur autorisé représentera l'étape principale de la diffusion de la carte. Le médecin qui remplira le formulaire requis et fournira les documents nécessaires obtiendra sa nouvelle attestation de professionnel par courrier en quelques jours ou semaines. Comme la CPS n'est pas directement délivrée par la FMH mais qu'elle doit l'être par un organisme dûment autorisé et 
surveillé, sa production impliquera des exigences élevées en matière de sécurité.

Le détenteur d'une CPS pourra utiliser sa carte en toute sécurité dans un environnement informatisé dit «ouvert», que ce soit avec son équipement existant ou en acquérant l'infrastructure nécessaire à un coût minimal. Dans cet

\section{E-Health - FMH}

Judith Wagner, responsable du domaine eHealth de la FMH

Le Conseil fédéral a adopté en juin 2007 une stratégie suisse de la cybersanté (eHealth), stratégie qui avait été élaborée conjointement par l'OFSP et les cantons. Un organe de coordination eHealth soutenu par la Confédération et les cantons a été créé en début d'année, afin d'appliquer cette stratégie. Cet organe de coordination doit élaborer des recommandations à l'attention de tous les acteurs de la santé. Il existe une volonté politique claire d'ancrer ces recommandations dans la législation, si le besoin s'en faisait sentir [1].

L'organe national de coordination est composé d'un secrétariat avec trois collaborateurs et de nombreux groupes de travail, dirigés par un comité de pilotage réunissant des représentants des cantons et de la Confédération, avec à sa tête le président de la Confédération Pascal Couchepin. De plus, un groupe d'accompagnement consultatif composé de représentants des divers groupes d'intérêts donne son avis avant les décisions importantes. Le travail technique est subdivisé en divers projets partiels dont certains peuvent fonctionner avec des sous-groupes. Le travail de ces projets partiels est coordonné par un comité de direction des projets et ils doivent appliquer un calendrier très ambitieux, une consultation sur les premières propositions de solution devant avoir lieu dès novembre.

La stratégie suisse de la cybersanté (eHealth) a été élaborée sans impliquer le corps médical, bien que les médecins soient, avec les patients, les principaux acteurs concernés. Il est d'autant plus important que la communauté médicale adopte une position claire dans ce domaine pour qu'elle puisse ensuite la faire sienne.

La FMH est convaincue que les projets E-Health ne peuvent être menés avec succès sans impliquer le corps médical, et représente donc le corps médical dans les différents comités en conséquence. environnement, le mot «ouvert» signifie que les documents pourront être choisis librement, indépendamment de leur qualité ou de leur origine, et qu'ils seront compatibles. Le soutien électronique à disposition garantira bien entendu une protection complète des données et un fonctionnement optimal de l'application.
La FMH insiste pour que les attentes soient et restent réalistes et que les technologies de l'information et de la communication ne soient pas utilisées pour modifier les habitudes, c'est-à-dire pour imposer des changements dans les processus de travail («do not use a computer to force a change of behaviour»).

La FMH défend donc par exemple les positions suivantes: les outils E-Health doivent permettre de créer les conditions pour un échange électronique d'informations, rien ne sert de tenter de standardiser les méthodes de travail des médecins, mais il vaut mieux harmoniser les processus d'échange. Le principal est d'aider à l'échange des données entre les divers acteurs des traitements, par exemple, le contenu des lettres de sortie n'a pas besoin d'être entièrement standardisé pour permettre cet échange. Par contre, l'expéditeur, le destinataire et les patients concernés doivent pouvoir être clairement identifiés.

Le corps médical tient particulièrement à ce que l'utilisation des outils E-Health reste facultative et qu'une protection complète des données soit assurée pour les patients et les médecins. Même à l'avenir et avec le E-Health, la confiance restera à la base de tout processus thérapeutique.

La FMH propose de mettre sur pied un groupe de travail interne E-Health, qui aidera à définir et coordonner nos prises de positions, en particulier par rapport à l'organe de coordination eHealth. Le positionnement général du corps médical étant essentiel, ce groupe devra également thématiser les contenus d'une stratégie E-Health de la FMH.

\section{Référence \\ 1 Voir à ce sujet: www.bag.admin.ch/themen/ krankenversicherung/04108/index.html?lang=fr}




\section{Carte de professionnel de santé (CPS): questions et réponses}

Qui peut obtenir une CPS? Quelles sont les conditions à remplir?

Tous les médecins exerçant en Suisse peuvent obtenir une CPS. Les membres de la FMH reçoivent automatiquement un formulaire de demande. Les médecins qui ne sont pas membres de la FMH doivent attester leurs qualifications. En outre, les personnes suivantes peuvent obtenir une CPS:

- les collaborateurs d'un médecin selon les indications de celui-ci;

- les collaborateurs d'une institution de santé (p.ex. hôpital) selon les indications de la direction concernée.

A combien s'élèvent les coûts d'une CPS?

La carte contenant un certificat simple pour le trafic avec myfmh est incluse dans la cotisation de membre FMH. Par l'intermédiaire de la FMH, des certificats pour une signature juridiquement valable peuvent être commandés au prix très avantageux de 20 à 50 CHF. En comparaison, les prix du marché pour des certificats qualifiés sont cinq à huit fois plus chers.

Quelles sont les conditions techniques à remplir pour pouvoir utiliser la CPS au cabinet médical?

Pour l'utilisation d'une CPS, il est nécessaire de disposer d'un lecteur de cartes standard, d'un logiciel adapté à la CPS et d'une application qui fonctionne avec les certificats standards (p.ex. Outlook, Acrobat Professional, etc.).

Des lecteurs de cartes standards sont inclus dans certains ordinateurs portables et fixes ou peuvent être obtenus sous forme d'option conjointement avec la CPS.

Un logiciel pilote (driver) pour la CPS peut être téléchargé en ligne ou est offert en option sur CD. Il est utilisable sur les supports suivants:

- Windows 2000, XP et Vista

- Mac OS X pour Intel / Power PC

- Au moins l'un des dérivés Linux suivants: Fedora/Red Hat, Ubuntu, Suse.

Pour l'utilisation de la CPS avec la carte d'assuré, on pourra obtenir en option un certificat permettant d'accéder à celle-ci.

Quel est le degré de sécurité de la CPS?

La CPS dispose du degré de sécurité actuellement le plus élevé; les puces cryptées de la CPS et les clés électroniques qu'elle utilise correspondent aux dispositions légales en matière de signatures électroniques, valables selon la loi suisse sur la signature, et elles sont attribuées à une personne clairement identifiée sur la base d'une procédure reconnue par la loi. Seules quatre entreprises certifiées remplissent actuellement les conditions légales pour la fabrication d'une telle CPS. En outre, les données électroniques peuvent être codées de façon à ne pouvoir être lues que par le destinataire ou la personne autorisée.

L'interopérabilité de la CPS-FHM avec les CPS d'autres pays et d'autres groupes professionnels est-elle garantie?

La CPS est basée sur des standards internationaux. Ceci garantit pour la CPS une interopérabilité dans le monde entier avec toutes les cartes avec signature également basées sur ces standards.

Puis-je, avec la CPS, transférer des données en toute sécurité à un participant HIN et vice-versa?

Il résulte des premiers tests qu'un participant HIN peut envoyer un courrier électronique muni d'une signature numérique à tout participant de son choix. L'inverse n'est pas encore possible. HIN a promis d'effectuer les adaptations nécessaires sur le «client ASAS» afin d'assurer l'interopérabilité avec la CPS.

Qu'offre la CPS en plus face aux services HIN?

La CPS permet d'élargir le cercle des participants à un échange de données sécurisé, lequel est possible dans le monde entier sans se limiter aux participants disposant de l'accès à HIN.

Grâce à la CPS, il est possible de simplifier cet échange et de le réaliser sur la base d'applications standards gratuites, et aussi d'accéder à des services de données qui ne seraient pas atteignables par une connexion HIN.

Enfin, la CPS rend possible la signature de données sous forme numérique. Le destinataire peut constater sans le moindre doute qui a signé les données et si celles-ci ont subi des modifications durant leur transfert.

La CPS me permet-elle d'ouvrir une session avec un hôpital?

Oui, si le système informatique de l'hôpital reconnaît le certificat CPS en question et autorise la communication. 
Puis-je, avec la CPS, signer électroniquement des documents de manière à ce que cette signature ait la même valeur légale qu'une signature manuelle?

Vous pouvez signer des documents électroniquement avec la CPS. Si un certificat qualifié y figure et qu'il est utilisé, cette signature a la même valeur légale qu'une signature manuelle.

Qu'est-ce qui différencient les certificats de la CPS des certificats disponibles gratuitement sur internet? Sur le plan technique, les certificats disponibles sur internet sont généralement des certificats standards semblables à ceux de la CPS et ils ont aussi une interopérabilité.

Les différences résident dans les processus d'enregistrement des détenteurs de certificat, dans l'attribution des clés cryptées à ces derniers et dans les mesures de sécurité réglant la fabrication et le dépôt desdites clés.

Pour la CPS, on a choisi un fabricant qui remplit les exigences de sécurité légales les plus élevées.
Puis-je utiliser la CPS conjointement avec un accès HIN?

La CPS peut en principe être utilisée sur chaque ordinateur, indépendamment de la connexion Internet, aussi longtemps que cette connexion transmet avec transparence les données. Cette condition n'est actuellement pas remplie par la connexion HIN, mais l'opérateur a garanti qu'il procéderait aux modifications nécessaires pour assurer cette interopérabilité.

Pourquoi produisons-nous une carte et non une clé USB, ce qui serait bien moins compliqué?

Une carte ou une clé USB peuvent en principe être utilisées tout aussi bien comme CPS électronique. En revanche, une CPS servant d'attestation visuelle est uniquement réalisable sous forme de carte. En outre, une clé cryptée est actuellement bien plus chère qu'une carte cryptée. C'est pourquoi la FMH s'est déterminée pour une carte. Une clé USB appropriée sera aussi disponible en temps utile sous forme d'option. 